\title{
A NECESSARY AND SUFFICIENT EIGENVECTOR CONDITION FOR A CONNECTED GRAPH TO BE BIPARTITE*
}

\author{
SEBASTIAN M. CIOABĂ ${ }^{\dagger}$
}

\begin{abstract}
We give a necessary and sufficient condition for a graph to be bipartite in terms of an eigenvector corresponding to the largest eigenvalue of the adjacency matrix of the graph.
\end{abstract}

Key words. Graph, Bipartite, Principal eigenvector, Independent set.

AMS subject classifications. 05C50, 15A18.

1. Main Result. The eigenvalues and eigenvectors of the adjacency matrix of a graph $G$ are closely related to many important properties of $G$ (see $[1,3,5]$ for example). It is known that a connected graph is regular if and only if the largest eigenvalue of its adjacency matrix equals the maximum degree (or the average degree) of the graph. This is also equivalent to the fact that the all ones vector is an eigenvector of the adjacency matrix of $G$. Another well known result in spectral graph theory states that a graph is bipartite if and only if the spectrum of its adjacency matrix is symmetric with respect to zero. For connected graphs, this is equivalent to the fact that the negative of the largest eigenvalue of the adjacency matrix is also an eigenvalue of the adjacency matrix (see [1] or [5] Exercise 19).

In this short note, we give a necessary and sufficient condition for a connected graph to be bipartite in terms of an eigenvector corresponding to its largest eigenvalue. Our result also extends and improves previous work from $[2,7]$.

For a connected graph $G=(V, E)$ of order $n$, let $\lambda_{1} \geq \lambda_{2} \geq \ldots \geq \lambda_{n}$ denote the eigenvalues of its adjacency matrix $A$. By the Perron-Frobenius Theorem (see $[1,3,4])$, we know that $\lambda_{1}$ has multiplicity one and there exists a positive eigenvector $x$ corresponding to $\lambda_{1}$ such that $\sum_{i \in V} x_{i}^{2}=1$. The eigenvector $x$ is called the principal eigenvector of $G$ in [2]. Our main result is stated in terms of this eigenvector.

* Received by the editors April 8, 2010 Accepted for publication July 13, 2010. Handling Editor: Stephen J. Kirkland.

${ }^{\dagger}$ Department of Mathematical Sciences, University of Delaware, Newark, DE 19716-2553, USA (cioaba@math.udel.edu). Research supported by a startup grant from the Department of Mathematical Sciences of the University of Delaware. 
THEOREM 1.1. If $S$ is an independent set of a connected graph $G$ and $x$ is the principal eigenvector of $G$, then

$$
\sum_{i \in S} x_{i}^{2} \leq \frac{1}{2}
$$

Equality happens if and only if $G$ is bipartite having $S$ as one color class.

Proof. For each $i \in S$, we have that $\lambda_{1} x_{i}=\sum_{j \sim i} x_{j}$. Multiplying this equation by $x_{i}$ and adding up over all $i \in S$, we get that

$$
\lambda_{1} \sum_{i \in S} x_{i}^{2}=\sum_{i \in S} \sum_{j \sim i} x_{i} x_{j} .
$$

Because $S$ is independent and the entries of $x$ are positive, we deduce that

$$
\sum_{i \in S} \sum_{j \sim i} x_{i} x_{j} \leq \sum_{k l \in E} x_{k} x_{l}=\frac{x^{t} A x}{2}=\frac{\lambda_{1}}{2} .
$$

As $\lambda_{1}>0$, these two relations imply the desired inequality.

Equality happens here if and only if for any edge $k l$ whose endpoints are not in $S$, we have that $x_{k} x_{l}=0$. Because the entries of $x$ are positive, this happens if and only if there are no edges with both endpoints in the complement of $S$. This means that $G$ is bipartite with $S$ as one color class and finishes the proof.

It is easy to convert this theorem into an algorithm to determine if a connected graph $G$ is bipartite. Let $u$ be a fixed vertex of $G$ and let $W$ denote the set of vertices at even distance from $u$. If there is an edge with two endpoints in $W$, then $G$ is not bipartite. Otherwise, $W$ is independent and by the previous theorem, $G$ is bipartite if and only if $\sum_{w \in W} x_{w}^{2}=\frac{1}{2}$. The only difference from this procedure and the usual combinatorial algorithm for bipartiteness is that instead of checking that there are no edges with both endpoints not in $W$, we compute $\sum_{w \in W} x_{w}^{2}$ and compare it with $\frac{1}{2}$.

We note here that the recent work [6] proving sufficient eigenvalue conditions for the existence of odd cycles in graphs is based on properties of principal eigenvectors.

It would be interesting to find relations between eigenvectors of graphs and other combinatorial invariants such as chromatic number or girth.

\section{REFERENCES}

[1] A.E. Brouwer and W.H. Haemers. Spectra of Graphs. Available at http://homepages.cwi.nl/ aeb/math/ipm.pdf

[2] S.M. Cioabă and D.A. Gregory. Principal eigenvectors of irregular graphs. Electron. J. Linear Algebra, 16:366-379, 2007. 
[3] C. Godsil and G. Royle. Algebraic Graph Theory. Graduate Texts in Mathematics, 207. SpringerVerlag, New York, 2001.

[4] R. Horn and C. Johnson. Matrix Analysis. Cambridge University Press, Cambridge, 1990.

[5] L. Lovász. Combinatorial Problems and Exercises. AMS Chelsea Publishing, 2007.

[6] V. Nikiforov. A spectral condition for odd cycles. Linear Algebra Appl., 428:1492-1498, 2008.

[7] B. Papendieck and P. Recht. On maximal entries in the principal eigenvector of graphs. Linear Algebra Appl., 310(1-3):129-138, 2000. 